\title{
Characterization of bulk piezoelectric element-based ultrasonic motors
}

\author{
Shunsuke Izuhara* (10) and Tomoaki Mashimo
}

\begin{abstract}
We propose miniature ultrasonic motors using stators made of a single bulk piezoelectric element. The bulk piezoelectric stator has the potential to increase output by reducing the dissipation that occurs in the adhesion layer between the PZTs and a metallic component in the stator. In this paper, we build two kinds of bulk piezoelectric stators: a cubic bulk stator with a side length of $4.2 \mathrm{~mm}$ and a hole of $3 \mathrm{~mm}$ in diameter, and a cylindrical bulk stator with an outer diameter of $4.2 \mathrm{~mm}$ and the same hole diameter. We evaluate their electrical and mechanical characteristics, such as impedance and vibration velocity. The experiments clarify the advantages of the bulk piezoelectric stators, such as low dissipation. The cubic bulk stator, which shows higher performance than the cylindrical one, is compared with a similar-shaped stator using a bronze cube and thin piezoelectric plates. The performance measures of the cubic bulk ultrasonic motor are optimized by the preload between the stator and rotor.
\end{abstract}

Keywords: Piezoelectric actuators, Ultrasonic motors, Micromotors, PZT

\section{Introduction}

Millimeter-scale electric motors with a high torque density are one of the essential components in realizing future mechatronic devices in mobiles, medicine, and rescue. Many micromotors have been proposed in the past 30 years [1-4]. Electromagnetic motors are the most available actuators but have several problems for further miniaturization. Not only they require tiny components, but they also induce serious torque reduction due to the scaling law, in which the torque scales down with the fourth power of a characteristic length $[5,6]$. Very small actuators less than sub-millimeters use electrostatic forces. Electrostatic actuators have excellent scalability and enable their miniaturization by MEMS process [7, 8], but their weak driving force limits their deployment as millimeter-scale motors.

Piezoelectric ultrasonic motors should be the best actuators in the middle scale, ranging from submillimeter to millimeters, where both electromagnetic and electrostatic principles are difficult to apply [9]. Ultrasonic

*Correspondence: izuhara.shunsuke.gk@tut.jp

Toyohashi University of Technology, 1-1 Hibarigaoka Tenpakucho,

Toyohashi 441-8580, Japan motors are known as one of the prominent miniature motors because of their high torque density and simplicity [10-12]. Miniature ultrasonic motors of $4.5 \mathrm{~mm}$ have been implemented in watches [13], and those of 10 $\mathrm{mm}$ in camera lenses [14]. In all existing commerciallyused ultrasonic motors, an annular piezoelectric plate is bonded to a metallic ring by adhesives . This metallic ring plays an important role in enlarging the vibration amplitude because the original amplitude of piezoelectric ceramics is very small. (The strain of the piezoelectric ceramics is known as roughly $0.1 \%$, no matter how high the applied voltage is). However, when miniaturizing ultrasonic motors, we want to avoid using the metallic component for two negative reasons. First, highquality fabrication of the metallic ring is difficult at small scale. For example, a trivial burr that can be ignored at macro scale becomes a relatively large burr that must be removed in microscale. Second, the mechanical dissipation in the adhesion layer between the metal and piezoelectric element increases when the size becomes small [15].

A solution to those problems is the use of a stator consisting of a single bulk piezoelectric element without any metallic component. This idea simplifies the mechanical 
design and reduces the fabrication cost. It has the possibility of reducing mechanical dissipation and improves the motor performance measures. There have been several studies on building miniature ultrasonic motors by a bulk piezoelectric element. The first study on using a bulk piezoelectric stator suggested building a cylindrical ultrasonic motor with a diameter of $2.4 \mathrm{~mm}$ and a length of 10 $\mathrm{mm}$ [16]. It showed a relatively high output but requires complicated fabrication, such as micromachining for thin piezoceramic tubes. After that, building bulk stators was attempted for smaller ultrasonic motors, such as a cylindrical stator with a diameter of $2 \mathrm{~mm}$ and $6 \mathrm{~mm}$ [17]. However, they require a difficult fabrication process for high aspect ratio. Most recently, a flat bulk piezoelectric stator with a diameter of $4.2 \mathrm{~mm}$ fabricated by MEMS is suggested [18]. It showed the performance measures of the prototype motors, but the resultant torque was still small.

In this paper, we propose miniature ultrasonic motors using stators made of a single bulk piezoelectric element. Two examples of bulk stators are built for evaluation: a cubic bulk stator with a center hole and a hollow cylindrical bulk stator. They are compared to a similar-sized stator using a metallic component experimentally. The comparison clarifies how bulk stators are advantageous for use in ultrasonic motors. Finally, the performance measures of the cubic bulk ultrasonic motor are evaluated and compared with that of using a metallic component. This paper is organized as follows. In section "Driving principle and FEM piezoelectric analysis", the driving principle and the FEM piezoelectric analysis are explained. Section "Vibration analysis" compares the cubic bulk stator, cylindrical bulk stator, and the cubic metallic stator. In section "Evaluation of a bulk piezoelectric ultrasonic motor", the motor performance using a cubic bulk stator is evaluated.

\section{Driving principle and FEM piezoelectric analysis Driving principle}

As the driving principle for miniature ultrasonic motors, a first-order bending vibration mode of a hollow cylindrical stator is often used [19-21]. The bending mode peaks the vibration velocity at the end of a cylinder. The vibration amplitude of the bending mode is larger than that of other modes and obtains a beneficial effect on the motor performance, but the amplitude decreases severely when the cylinder length is shorter. In this paper, we use the three-wave mode that generates three waves around the circumference of the stator hole as a driving principle. It is expected as one of the most prominent miniature motors because of the simple fabrication process and high energy density [22]. Unlike the bending mode, the ultrasonic motors driven by the three-wave mode can generate a certain torque when the stator is fabricated as cubic or flat. This characteristic makes the fabrication easy and is suited for the miniaturization.

Let us explain the driving principle of the bulk piezoelectric element-based ultrasonic motor briefly. Figure 1(a) shows the first vibration mode exciting three waves along the circumference of a hole. Figure 1(b) shows the second three-wave mode with a wavelength difference of $\pi / 2$ along the circumference. When the two vibration modes have a temporal phase difference of $\pi / 2$, the stator produces a traveling wave around the circumference of the stator hole. The surface of this traveling wave generates an elliptical motion, as with the driving principle of typical traveling wave ultrasonic motors [23-25]. This elliptical motion transfers its driving force to the rotor inserted into the hole via friction, spinning the rotor.

\section{FEM piezoelectric analysis}

We examine whether the bulk piezoelectric stator generates the three-wave mode inside its hole in finite element method (FEM) piezoelectric analysis (FEMTET, Murata software co., Tokyo, Japan). Let us consider a bulk stator with a cubic shape and a center hole. The cubic bulk stator is modeled in the FEM piezoelectric analysis. The polarization direction is the radial direction from the inside of the hole to the outside of the cube, as shown in Fig. 2a: The external four surfaces are positive and the one internal surface is negative. The four electrodes are placed on the four side surfaces of the stator, respectively, and one curved electrode is located on the inner surface of the stator hole, as shown in Fig. 2b. The material parameters are those of the piezoelectric material used in the experimental sections. In the modal analysis/resonant analysis, the voltage $e=A_{E} \sin \left(2 \pi f_{E} t\right)$ is applied to the top electrode and the bottom electrode is connected to ground $(0 \mathrm{~V})$. When the voltage frequency $f_{E}$ equals the resonant frequency of the three-wave mode, the three-wave mode appears in the simulation results. Figure $2 \mathrm{c}$ shows the result of analysis. The cubic bulk stator

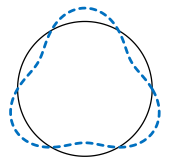

a

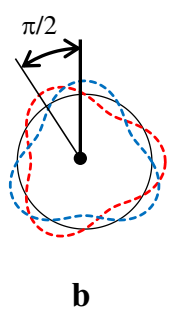

Fig. 1 a Vibration mode exciting three waves along the circumference of a hole in stator. (a) The first three-wave mode and (b) the second three-wave modes with a wavelength difference of $\pi / 2$ 


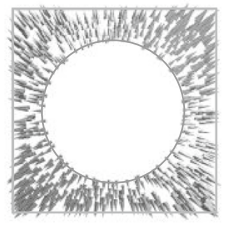

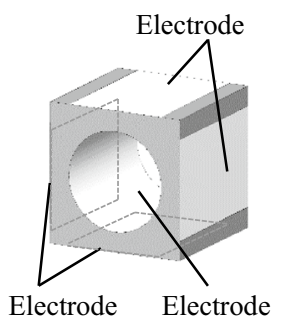

b

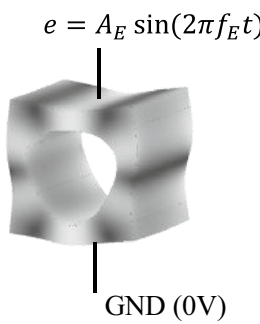

c

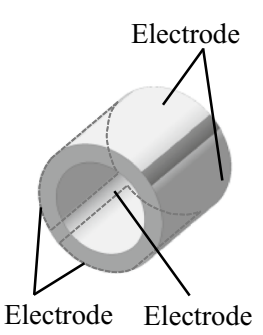

d

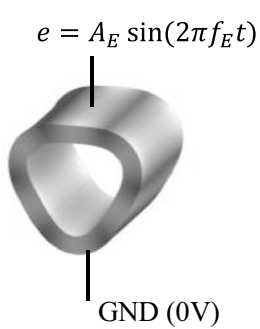

e

Fig. 2 a Polarization direction of the bulk piezoelectric stator in piezoelectric analysis model. b Electrodes placed on the bulk stator, c three-wave mode generated in the bulk piezoelectric stator when a voltage is applied. $\mathbf{d}$ The model of the cylindrical bulk stator, and $\mathbf{e}$ the three-wave mode on the cylindrical bulk stator

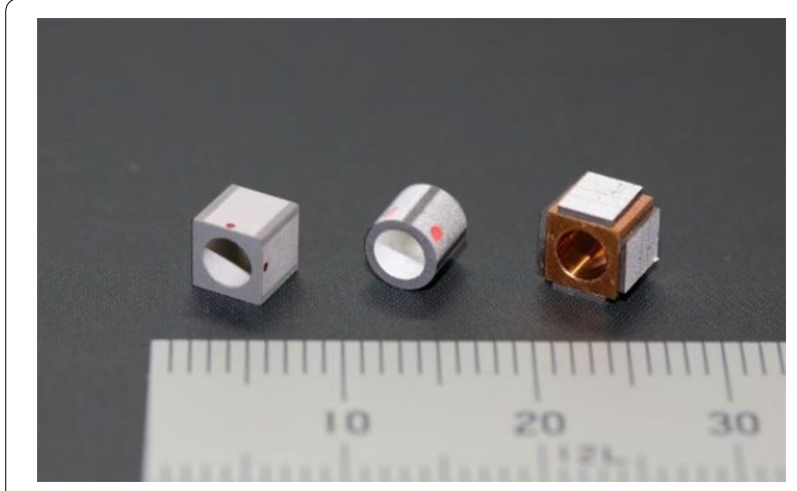

Fig. 3 Prototype stators. From the left, the cubic piezoelectric bulk stator, the cylindrical piezoelectric bulk stator, and the conventional stator comprising of a bronze cube and the thin piezoelectric plates bonded to the cube

can generate a three-wave mode around its hole in the proposed designs.

Another design of the bulk stator is a hollow cylinder, the shape of which can be fabricated by ordinary machining processes. The difference between the two designs is the existence of four corners. For the resonant analysis, the polarization direction and material properties are defined in the FEM model of the hollow cylindrical bulk stator (Figure 2d). As with the cubic bulk stators, the three-wave mode is excited inside the hole of a cylindrical bulk stator (Figure 2e). The resulting resonant frequencies in both models are different because of the existence of the corners. The inductance of the cylindrical bulk stator decreases by the reduction of the mass of the corners and results in a smaller natural frequency of a hollow cylindrical one.

\section{Vibration analysis}

In this section, we evaluate the three stators shown in Fig. 3. The left and the center are bulk piezoelectric stators, and the right is a conventional stator comprising of a bronze cube with a hole and thin piezoelectric plates bonded to the cube. The bulk stators with cubic and cylindrical shapes are evaluated by an impedance analysis and a laser Doppler velocimetry. Comparison with the metallic stator shows the advantages of the bulk piezoelectric stators. We compare the two bulk stators with the cubic bronze stator by measuring the current and velocity.

\section{Prototype of the stators}

The bulk piezoelectric stators are produced by cutting and grinding processes, similar to those used for machinnig glasses and silicones. The hole at the stator center is cut open by a curve generator. After the shapes are formed, the inside electrode is made by dipping into a silver liquid solution, and four outside electrodes are printed. The bulk stators are polarized between four outside electrodes and one inner surface inside the hole.

The prototype cubic bulk stator measures a side length of $4.2 \mathrm{~mm}$ and a through-hole with a diameter of $3 \mathrm{~mm}$. The cylindrical bulk stator has an outer diameter of 4.2 $\mathrm{mm}$ and an inner diameter of $3 \mathrm{~mm}$. The material is a hard piezoelectric material with a high-quality factor (C-213, Fuji ceramics, Fujinomiya, Japan). The comparable metallic stator consists of a bronze cube with a side length of $4.2 \mathrm{~mm}$ and a hole of $3 \mathrm{~mm}$ in diameter and four piezoelectric plates with a surface of $4 \mathrm{~mm} \times 3 \mathrm{~mm}$ and a thickness of $0.5 \mathrm{~mm}$. The piezoelectric plates are bonded to the four side surfaces of the bronze cube using an epoxy adhesive.

Giving the design dimensions and material parameters to the FEM piezoelectric analyses, the mode shape is depicted with the resonant frequency of the three-wave mode. The resulting modal analyses show a three-wave mode of the cubic bulk stator at a frequency of $226 \mathrm{kHz}$, that of the cylindrical bulk stator at $209 \mathrm{kHz}$, and that of using the bronze cube at $252 \mathrm{kHz}$. 


\section{Impedance analysis}

An impedance analysis shows the resonance by the threewave mode and clarifies the electric characteristics. In this measurement, the top and bottom piezoelectric elements are connected to the signal and ground lines of the impedance analyzer (FRA5087, NF Corp., Yokohama, Japan), respectively. The current consumed at the stators $i$ is calculated from an impedance $z$, a phase $\theta$ and an applied voltage $e$ :

$$
i=\frac{e}{z} \cos \theta
$$

Fig. 4 shows the current consumed at the three stators when the amplitude of $10 \mathrm{~V}_{\mathrm{p}-\mathrm{p}}$ is constant and the frequency varies from 200 to $250 \mathrm{kHz}$. The cubic bulk stator resonates and increases the current at approximately 229 $\mathrm{kHz}$, which is almost the same as the resonant frequency predicted by the piezoelectric analysis. The current supplied to the cylindrical bulk stator peaks at about 207 $\mathrm{kHz}$, which is also similar to the prediction of piezoelectric analysis. Comparing the two currents under the same applied voltage, the cubic bulk stator supplies a larger current than the cylindrical one. This fact means that the cubic bulk stator can drive by a lower applied voltage. Compared to the bronze stator, the bulk stators can flow a larger current. This is because the impedance of the bronze cube stator is larger by the dissipation between the piezoelectric plates and metallic cube.

\section{Laser doppler velocity measurement}

The generation of the three-wave mode can be found by measuring the vibration velocity inside the stator hole. A laser Doppler vibrometer (NLV2500-5, Polytec Japan) measure the velocity of the surface without contact. Figure 5 shows the experimental setup for measuring the velocity of the stator. Voltage $e=A_{E} \sin \left(2 \pi f_{E} t\right)$

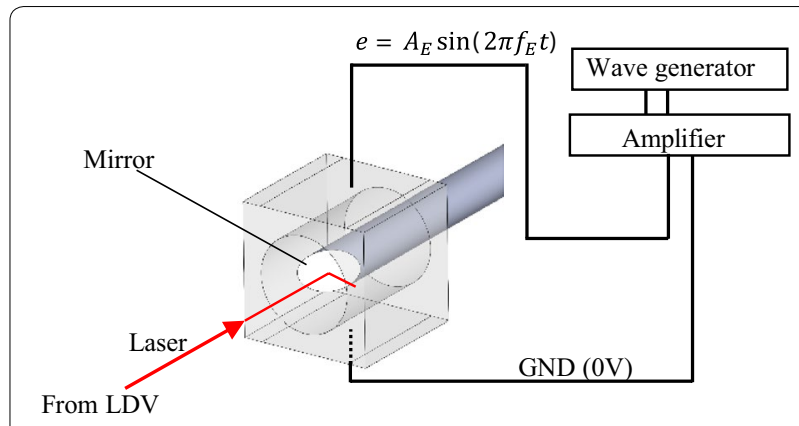

Fig. 5 Experimental setup for laser Doppler velocimetry

is applied to the top piezoelectric element and a ground line connects to the bottom one to be the same as the impedance analysis. The laser Doppler vibrometer emits a laser from the sensor head to the stator. A $45^{\circ}$ rod mirror is installed into the stator hole and it reflects the laser orthogonally to the inner surface of the hole. By rotating the mirror circumferentially, the nodes and antinodes can be observed. While the mirror rotates around in a complete circle, six nodes and antinodes are shown, and the number of the antinodes is accorded to that of the three-wave mode. The peak velocity obtained at the antinode of the three-wave mode is measured. Figure 6 shows the vibration velocity at inside the hole of the three stators when the amplitude of $A_{E}=10 \mathrm{~V}_{\mathrm{p}-\mathrm{p}}$ is constant and the frequency varies from $200 \mathrm{kHz}$ to $250 \mathrm{kHz}$, the same as those of impedance analysis. The peak velocity of the cubic bulk stator is higher than that of the cylindrical one at a given constant voltage. The velocity of the bronze cube stator is small and the bulk stators generate larger vibration velocities under the same applied voltage. In all, the frequencies of the peak velocity are accorded to the frequency of the peak current.
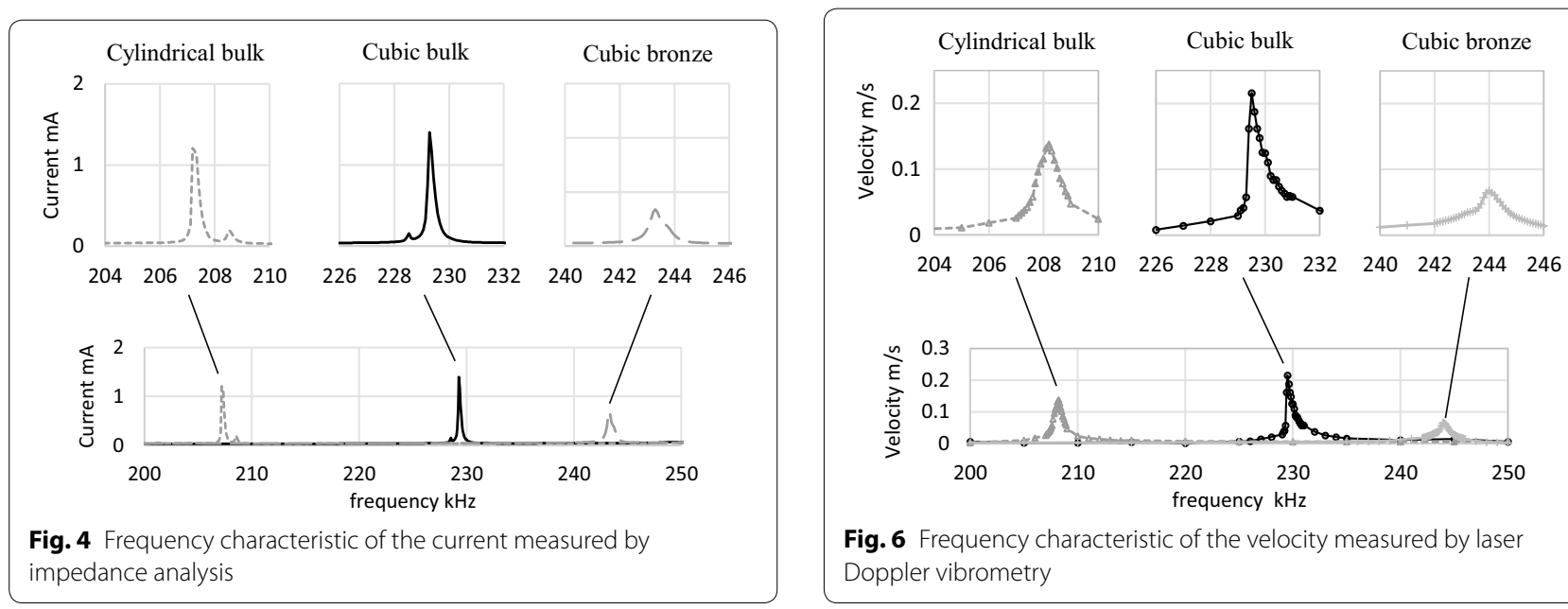

Fig. 6 Frequency characteristic of the velocity measured by laser Doppler vibrometry 


\section{Comparison of the stators}

The most well-used parameters for evaluating piezoelectric actuators are quality factor $Q_{m}$ and coupling coefficient $k$. The quality factor $Q_{m}$ expresses how underdamped the stator is and the sharpness of the supplied current at the resonance. It is calculated from the curve of current by the full width at half maximum method:

$$
Q_{m}=\frac{f_{0}}{f_{2}-f_{1}},
$$

where $f_{0}$ is the frequency that maximizes the current and the denominator $f_{2}-f_{1}$ is half-width of the current curve. The coupling coefficient $k$ means the ratio of the consumed mechanical energy to the supplied electric energy; that is, a stator with a higher coupling coefficient can drive with a lower voltage. It can be approximately estimated from the relationship between resonance and anti-resonance in the impedance curve [26]:

$$
k^{2} \simeq \frac{f_{a}^{2}-f_{r}^{2}}{f_{a}^{2}},
$$

where $f_{r}$ and $f_{a}$ are resonant and anti-resonant frequencies, respectively. The quality factor and coupling factor are obtained by the impedance analysis.

The behavior of the current is ideally analogical to that of the velocity, but there is a difference between the ideal and the actual-the quality factor $Q_{m}$ obtained by the laser Doppler velocimetry differs from that obtained by the impedance analyzer in each stator. To show this difference, the quality factor obtained by the laser Doppler velocimetry should be evaluated, but measuring the quality factor of the velocity is difficult because the frequency response of the velocity depends on the non-linearity of PZTs. Instead of that, we define the current-velocity ratio $A_{i v}$ obtained from the peak current $i_{\text {peak }}$ and the peak velocity $v_{\text {peak }}$. The values of $i_{\text {peak }}$ and $v_{\text {peak }}$ are obtained in the experiments at previous subsections. The current-velocity ratio is expressed as

$$
A_{\text {iv }} \triangleq \frac{i_{\text {peak }}}{v_{\text {peak }}} .
$$

This equation shows that the smaller current-velocity ratio obtains a higher vibration velocity under a unit current consumption.

Table 1 summarizes the quality factor $Q_{m}$, coupling coefficient $k$, and the current-velocity ratio $A_{i v}$. The cubic bulk stator obtains the highest quality factor: it means the existence of corners increases the vibration with low dissipation in the comparison with the
Table 1 Performance measures of stators

\begin{tabular}{llll}
\hline Stator type & $\begin{array}{l}\text { Quality } \\
\text { factor } \boldsymbol{Q}_{\boldsymbol{m}}\end{array}$ & $\begin{array}{l}\text { Coupling } \\
\text { coefficient } \boldsymbol{k} \%\end{array}$ & $\boldsymbol{i - \boldsymbol { v } \text { ratio } \boldsymbol { A } _ { \boldsymbol { i v } } \mathbf { A s } \mathbf { m }}$ \\
\hline Cubic bulk & 1524 & 6.52 & 0.0130 \\
Cylindrical bulk & 1134 & 6.03 & 0.0145 \\
Cubic bronze & 465 & 7.63 & 0.0201 \\
\hline
\end{tabular}

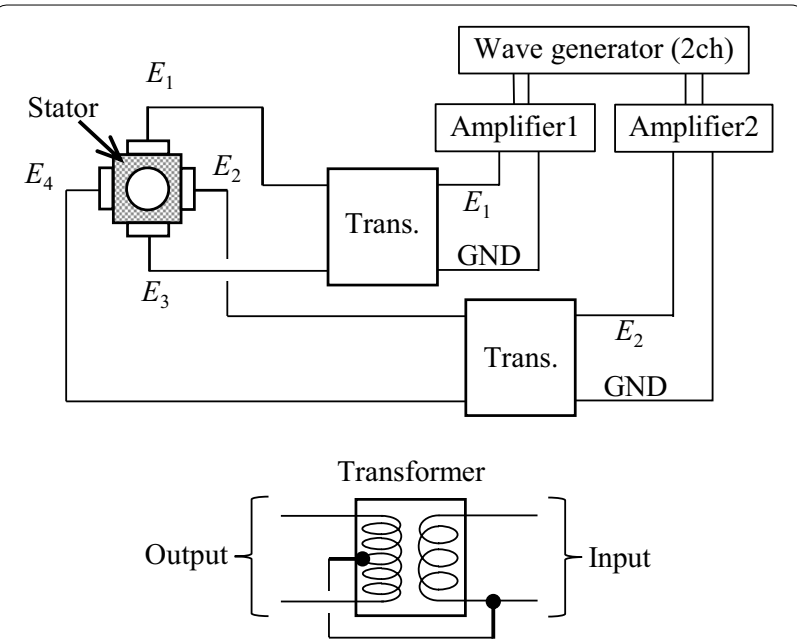

Fig. 7 Experimental setup to apply voltages to the stator

cylindrical one. The coupling factor is slightly lower, but the obtained quality factor is more than three times larger than the cubic bronze stator. The bulk stators obtain better current-velocity ratio than the cubic bronze stator. This is because the damping of the piezoelectric material is smaller than that of using an adhesive with a metallic component.

\section{Evaluation of a bulk piezoelectric ultrasonic motor}

The bulk piezoelectric stators show better characteristics compared to the bronze stator with adhesion. However, the motor performance of using bulk stators is still unclear because other factors might influence on the performance. In this section, we evaluate the ultrasonic motor using the bulk stator and compare it with that using the bronze cube stator by experiments.

\section{Experimental setup}

Figure 7 shows how to apply the voltages to the stator. To drive the by the three-wave mode, two different voltages, $E_{1}$ and $E_{2}$ with a voltage amplitude $A_{E}$ and a frequency $f_{E}$, are applied to the piezoelectric elements on the stator. The frequency $f_{E}$ is adjusted to the resonant frequency that excites the three-wave mode in the stator. When the first voltage $E_{1}=A_{E} \sin \left(2 \pi f_{E} t\right)$ 
is applied to the top piezoelectric element, the stator excites a three-wave mode. When the second voltage $E_{2}=A_{E} \cos \left(2 \pi f_{E} t\right)$ is applied to the right piezoelectric element, another three-wave mode is excited. The voltages $E_{1}$ and $E_{2}$ have a temporal phase difference of $\pi / 2$ while two three-wave modes present a wavelength difference of $\pi / 2$.To enlarge the vibration, the four piezoelectric elements are composed at the four sides of the stator and the four voltages are applied. Besides, two voltages $E_{3}$ and $E_{4}$ are applied to the bottom and left surfaces. They have a reverse phase from $E_{1}$ and $E_{2}$, respectively: $E_{3}=-A_{E} \sin \left(2 \pi f_{E} t\right)$ and $E_{4}=-A_{E} \cos \left(2 \pi f_{E} t\right)$.

Two power supplies and transformers are used to generate the four voltages $E_{1}-E_{4}$. Two high-frequency voltages are generated using a wave generator (WF1974, NF Corp., Yokohama, Japan) and amplified by power amplifiers (HSA4052, NF Corp.). In addition to the amplified voltages, transformers generate voltages with reversed phases. For example, in a power supply line, when voltage $E_{1}$ with sine phase is input to a transformer, another voltage $E_{3}$ with a negative sine phase is output from a transformer terminal. Thus, two voltages $E_{1}$ and $E_{3}$ are obtained from two terminals of a transformer. Through transformers, voltages $E_{1}-E_{4}$ are applied to the electrodes of the stator. The amplitudes, frequencies, and phase differences of these voltages can be adjusted using the wave generator and amplifiers.

When the voltages are applied, the rotor starts spinning. The rotor spin from the transient state to the steady-state is captured using a high-speed camera (VW9000, Keyence Co., Tokyo, Japan). The angular velocity and the angular acceleration of the rotor spin are computed from the changes in the still images obtained from the high-speed camera by using image-processing software. By tracking the movement of the marker on the rotor in subsequent images, time-history data of the angular displacement are accumulated in memory. The angular velocity and the angular acceleration are calculated by differentiating these data with time. The output torque is estimated from the angular acceleration and the moment of inertia.

The driving principle requires excitation of two threewave modes at the same driving frequency. The frequency of the three-wave mode by $E_{1}$ and $E_{3}$ (Line A) should be accorded to that by $E_{2}$ and $E_{4}$ (Line B) (Fig. 7). The discordance between the two modes occurs by several empirical reasons such as a fabrication quality and lowers the motor performance. For a fair comparison, the two three-wave modes excited in the cubic bulk and bronze stator are shown in Fig. 8a, b, respectively. Both show a good agreement of the three-wave modes between the lines A and B, although the behavior of the bronze stator is relatively gradual.

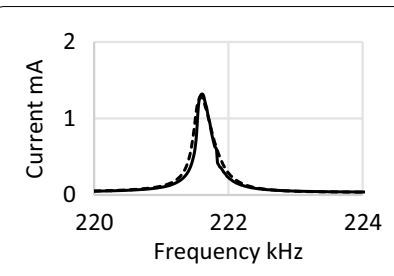

a b

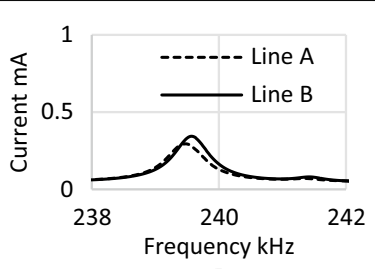

Fig. 8 Frequency characteristics of the current: a cubic bulk stator and $\mathbf{b}$ cubic bronze stator. Lines A and B are well accorded in the two figures

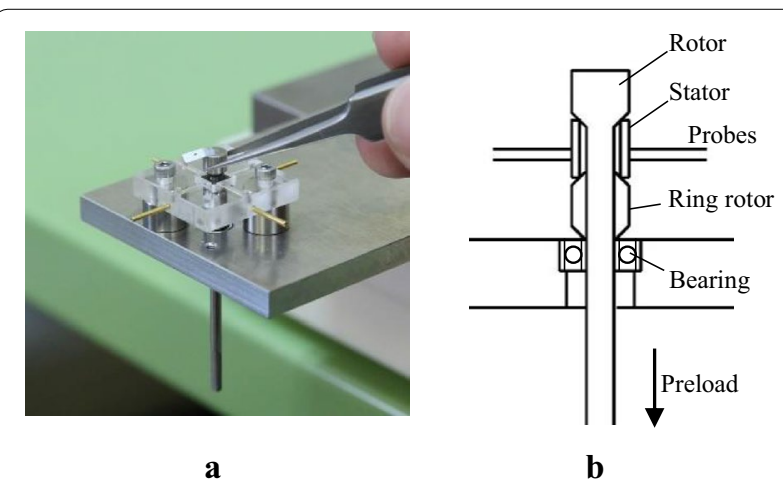

Fig. 9 Experimental setup for evaluating the motor performance measures, such as an angular velocity and torque

\section{Transient response}

We compare the transient response of the ultrasonic motors using the cubic bulk stator and the bronze stator. Figure 9a shows the experimental setup for measuring the transient response. The stator is supported by contact probes serving as connectors for applying voltages. As shown in the cross-section view (Fig. 9b), a rotor inserted to the stator contacts the upper edge of the stator hole and a hollow ring rotor contacts the bottom edge. Another side of the ring rotor contacts a bearing to reduce friction.

The angular velocity is obtained from the high-speed camera measurement. The high-speed camera tracks the movement of the marker on the rotor top (shown in Fig. 9a). Torque is estimated from the angular acceleration and the moment of inertia. (The moment of inertia of the rotor is $1.34 \times 10^{-9} \mathrm{kgm}^{2}$ ). Figure 10 shows the transient response of the rotor's angular velocity produced by the cubic bulk stator and the cubic bronze stator when a voltage amplitude of $30 \mathrm{~V}_{\mathrm{p}-\mathrm{p}}$ is applied to the two stators. The peak angular velocity of the cubic bulk reaches approximately $998 \mathrm{rpm}$, while that of the cubic bronze stator is less than $400 \mathrm{rpm}$. The output torque of approximately $15 \mu \mathrm{Nm}$ has been obtained in the cubic bulk stator. 


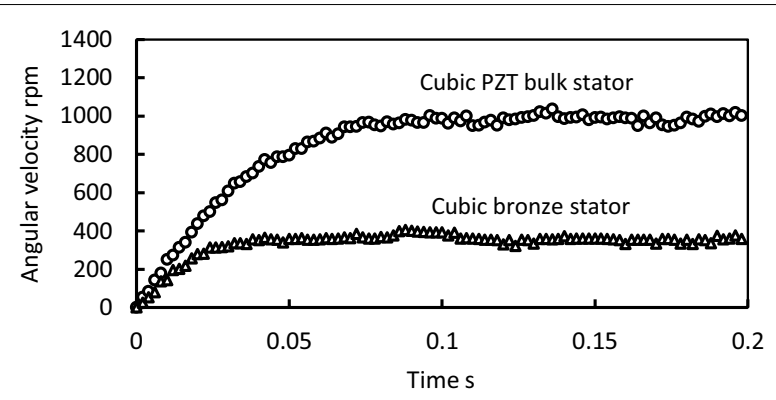

Fig. 10 Transient response of ultrasonic motors driven by the cubic bulk stator and the bronze cubic stator

Relationship between the preload and the motor output The torque of traveling wave ultrasonic motors improves with the increase in the preload between the stator and rotor. An interest is that the ultrasonic motors using the bulk stator also can generate a larger torque by the increase in the preload. The preload can be applied at the bottom of the rotor shaft as shown in Fig. 9b. When weights are applied to the rotor axially, the upper and bottom rotors sandwich the stator. By changing the number of the weights, the preload that acts at both edges of the stator can be adjusted. Several weights are attached to the rotor using a bearing that separates the motion of the rotor and weights. That is, the weights are stationary while the rotor spins, and the moment of inertia stays constant regardless of the weights.

We examine the optimal preload value that enhances the torque of the cubic bulk ultrasonic motor. The angular velocity is measured at the steady state of the rotation. The torque is computed by the second-order derivative of angular displacement. Because it makes errors large, the values of the torque are averaged for accurate evaluation. Figure 11 shows the angular velocity and torque when the preload varies under a constant voltage amplitude of $A_{E}=30 \mathrm{~V}_{\mathrm{p}-\mathrm{p}}$. (The rotor starts to spin from $20 \mathrm{~V}_{\mathrm{p}-\mathrm{p}}$ or less, but the angular velocity at the low voltage amplitude is unstable.) During this measurement, the voltage

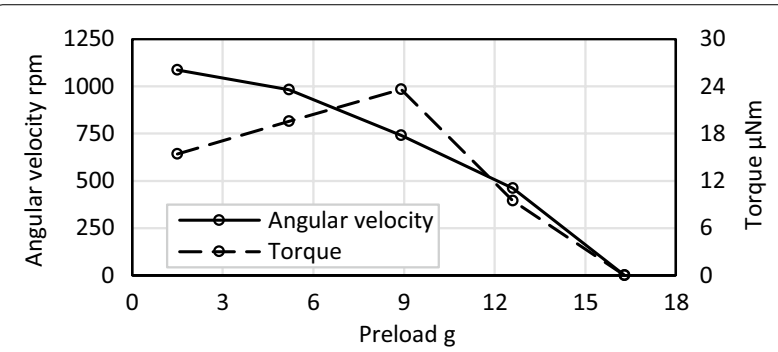

Fig. 11 Behavior of the angular velocity and the torque generated by the ultrasonic motor using the cubic bulk stator when the preload changes frequency $f_{E}$ is empirically adjusted to maximize the angular velocity and torque. For example, at a preload of $8.9 \mathrm{~g}$, the angular velocity peaks at $222.5 \mathrm{kHz}$ and the torque peaks at $223.5 \mathrm{kHz}$. The resulting angular velocity gradually decreases at larger preloads. This is because a large preload decreases the vibration amplitude of the stator and results in a decrease in the angular velocity. On the other hand, the torque increases with the preload and measures a peak torque of $24 \mu \mathrm{Nm}$ at a preload of 8.9 g. A too-large preload decreases the torque, and no rotation is obtained over $N=16 \mathrm{~g}$.

Figure 12 shows the relationship between the applied voltage and the motor output under a constant preload of $8.9 \mathrm{~g}$. The angular velocity and the torque increase at higher voltage amplitudes. A maximum angular velocity of $1250 \mathrm{rpm}$ and a maximum torque of $50.2 \mu \mathrm{Nm}$ are obtained at $120 \mathrm{~V}_{\mathrm{p}-\mathrm{p}}$.

\section{Conclusion}

The bulk stators for millimeter-scale ultrasonic motors were designed by FEM piezoelectric analyses and fabricated from bulk piezoelectric material. The resulting prototype stators reduced the mechanical dissipation and improved the performance measures in comparison to the stator using a metallic component and adhesives. The motor torque over $50 \mu \mathrm{Nm}$ is obtained and is much larger than those of the comparable bulk stators [16-18], although the size of the proposed stator is larger. As with the bottom-up process such as MEMS, the miniaturization process such as the fabrication of bulk piezoelectric ceramics also has potential and is an option as actuators for the miniature mechatronic devices. Because the mechanical components and adhesion are unnecessary, the bulk stators might be suited for mass production.

The improvement of the bulk piezoelectric ultrasonic motor would be started. There are several important issues to be clarified to improve the motor performance. For example, the optimization of the stator-rotor interface, such as coating the contact surface of the stator to obtain better friction and reduce wear. When the contact

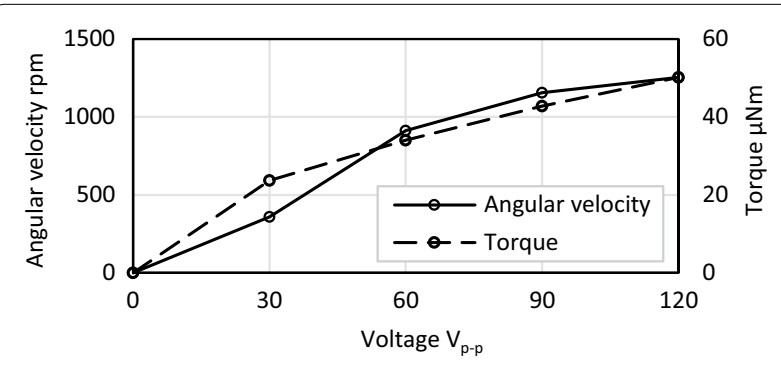

Fig. 12 Relationship between the voltage amplitude and the motor output under a constant preload of $8.9 \mathrm{~g}$ 
is optimized, the motor should perform with larger outputs at higher preload and higher voltage amplitude, as with the common ultrasonic motors.

\section{Acknowledgements}

This research has been supported in part by JSPS KAKENHI Grant Number $19 \mathrm{H} 02110$.

\section{Authors' contributions}

Authors' contributions All authors equally contributed. All authors read and approved the final manuscript.

\section{Funding}

This research has been supported in part by JSPS KAKENHI Grant Number $19 \mathrm{H} 02110$

\section{Availability of data and materials}

Not applicable.

Ethics approval and consent to participate

Not applicable.

\section{Consent for publication}

Not applicable.

\section{Competing interests}

The authors declare that they have no competing interests.

Received: 9 March 2020 Accepted: 7 August 2020

Published online: 19 August 2020

\section{References}

1. Ishihara H, Arai F, Fukuda T (1996) Micro mechatronics and micro actuators. IEEE/ASME Transact Mechatron 1(1):68-79

2. Watson B, Friend J, Yeo L (2009) Piezoelectric ultrasonic micro/milli-scale actuators. Sens Actuators A Phys 152(2):219-233

3. Liu DK-C, Friend J, Yeo L (2010) A brief review of actuation at the microscale using electrostatics, electromagnetics and piezoelectric ultrasonics. Acoust Sci Technol 31(2):115-123

4. Dong S (2012) Review on piezoelectric, ultrasonic, and magnetoelectric actuators. J Adv Dielectr 2(01):1230001

5. Trimmer WS (1989) Microrobots and micromechanical systems. Sens Actuators 19(3):267-287

6. Dermitzakis K, Carbajal JP, Marden JH (2011) Scaling laws in robotics. Procedia Comput Sci 7:250-252

7. Fan L-S, Tai Y-C, Muller RS (1989) Ic-processed electrostatic micromotors. Sens Actuators 20(1-2):41-47

8. Jacobsen S, Price R, Wood J, Rytting T, Rafaelof M (1989) A design overview of an eccentric-motion electrostatic microactuator (the wobble motor). Sens Actuators 20(1-2):1-16

9. Flynn AM, Tavrow LS, Bart SF, Brooks RA, Ehrlich DJ, Udayakumar KR, Cross LE (1990) Piezoelectric micromotors for microrobots. In: IEEE Symposium on Ultrasonics, pp 1163-1172.
10. Morita T (2003) Miniature piezoelectric motors. Sens Actuators A Phys 103(3):291-300

11. Uchino K, Cagatay S, Koc B, Dong S, Bouchilloux P, Strauss M (2004) Micro piezoelectric ultrasonic motors. J Electroceram 13(1-3):393-401

12. Peng $Y$, Peng $Y$, Gu X, Wang J, Yu H (2015) A review of long range piezoelectric motors using frequency leveraged method. Sens Actuators A Phys 235:240-255

13. lino A, Suzuki K, Kasuga M, Suzuki M, Yamanaka T (2000) Development of a self-oscillating ultrasonic micro-motor and its application to a watch. Ultrasonics 38(1-8):54-59

14. Nakagawa Y, Saito A, Maeno T (2008) Nonlinear dynamic analysis of traveling wave-type ultrasonic motors. IEEE Transactions Ultrasonics Ferroelectrics Frequency Control 55(3):717-725

15. Mashimo T (2017) Scaling of piezoelectric ultrasonic motors at submillimeter range. IEEE/ASME Transact Mechatron 22(3):1238-1246

16. Morita T, Kurosawa MK, Higuchi T (1999) Cylindrical micro ultrasonic motor utilizing bulk lead zirconate titanate (pzt). Japanese J Appl Phys 38(55):3347

17. Kanda T, Makino A, Ono T, Suzumori K, Morita T, Kurosawa MK (2006) A micro ultrasonic motor using a micro-machined cylindrical bulk pzt transducer. Sens Actuators A Phys 127(1):131-138

18. Hareesh P, DeVoe DL (2018) Miniature bulk pzt traveling wave ultrasonic motors for low-speed high-torque rotary actuation. J Microelectromech Syst 27(3):547-554

19. Kurosawa M, Nakamura K, Okamoto T, Ueha S (1989) An ultrasonic motor using bending vibrations of a short cylinder. IEEE Transact Ultrasonics Ferroelectrics Frequency control 36(5):517-521

20. Morita T, Kurosawa MK, Higuchi T (2000) A cylindrical shaped micro ultrasonic motor utilizing pzt thin film (1.4 mm in diameter and $5.0 \mathrm{~mm}$ long stator transducer). Sens Actuators A Phys 83(1-3):225-230

21. Yang L, Ren W, Ma C, Chen L (2020) Mechanical simulation and contact analysis of the hybrid longitudinal-torsional ultrasonic motor. Ultrasonics 100:105982

22. Mashimo T (2014) Micro ultrasonic motor using a one cubic millimeter stator. Sens Actuators A Phys 213:102-107

23. Sashida T, Kenjo T (1993) An Introduction to Ultrasonic Motors. Clarendon Press, Oxford

24. Renteria-Marquez I, Renteria-Marquez A, Tseng B (2018) A novel contact model of piezoelectric traveling wave rotary ultrasonic motors with the finite volume method. Ultrasonics 90:5-17

25. Zhou Y, Chang J, Liao X, Feng Z (2020) Ring-shaped traveling wave ultrasonic motor for high-output power density with suspension stator. Ultrasonics 102:106040

26. Nakamura K, Sasaki H, Shimizu H (1981) A piezoelectric composite resonator consisting of a zno film on an anisotropically etched silicon substrate. Japanese J Appl Phys 20(3):111

\section{Publisher's Note}

Springer Nature remains neutral with regard to jurisdictional claims in published maps and institutional affiliations.

\section{Submit your manuscript to a SpringerOpen ${ }^{\circ}$ journal and benefit from:}

- Convenient online submission

$\checkmark$ Rigorous peer review

- Open access: articles freely available online

- High visibility within the field

- Retaining the copyright to your article

Submit your next manuscript at $\boldsymbol{\nabla}$ springeropen.com 